\title{
A Generation Method of Dithering Signal Based on DFT
}

\author{
Changqing Ye, Xingzhong Xiong \\ Sichuan University of Science and Engineering, Zigong, China \\ Email:1972337846@qq.com,xzxiong@suse.edu.cn
}

How to cite this paper: Ye, C.Q. and Xiong, X.Z. (2017) A Generation Method of Dithering Signal Based on DFT. Int. J. Communications, Network and System Sciences, 10, 299-306.

https://doi.org/10.4236/ijcns.2017.108B032

Received: July 16, 2017

Accepted: August 11, 2017

Published: August 14, 2017

\begin{abstract}
This paper proposes a generation method of dithering signal based on Discrete Fourier Transform (DFT), which is not only independent with the input signal but also can decrease the quantization error of the Analog-to-Digital Converter $(\mathrm{ADC})$. A detailed investigation of three typical dithering effects on the quantization error in ADC has been also presented in this paper, to highlight the advantages of the proposed reconstructed dithering signal. The simulation experiment and theoretical analysis illustrate that the reconstructed dithering signal based on DFT can improve the performance of ADC in comparison with traditional typical dithering signal.
\end{abstract}

\section{Keywords}

Dithering, ADC, DFT, Quantization Error

\section{Introduction}

The academic researches of dithering technology have gained multitudinous remarkable attention in ADC area before 1951. It is widely acknowledged that developing innovative dithering signals are worthwhile and significative. And a large number of researchers have made a lot of efforts to concentrate on this attractive topic.

In [1], Goodall found using dithering technology on video pulse code modulation (PCM) to decrease the quantization effects. Then Robert did further research on developing contour effects with noise. He proposed that dithering added to an input signal of the ADC and then subtracting the dithering prior to its quantization and it is the earliest theory of adding or subtracting dithering [2]. Before 1960s, dithering had been widely used and researched. The processes of using Rober's theory-add an analog noise prior the input signal of quantizer and then subtract the noise after the quantization have many different discoveries. 
Widrow clarified statistical independence of the quantization noise and the input signal can minimize losses of quantization [3]. Schuchman studied the effect of dithering on the quantization noise, and he gave a statistical independence condition of quantization noise and input signal [4]. Spang and Schultheiss found that dithering can change the frequency content of ADC' noise. In the processes of dithering added, although the noises of specified frequency are decreased, the total errors are increased [5]. Blesser proposed the concept of non-subtractive dithering [6] [7]. In 1984, Vanderkooy and Lipshitz found that dithering effect can improve the quantization resolution [8]. They analyze the application of dithering in videos from theoretical and experimental. They applied the research results to the audio signal and proved that dithering can transform the distortion signals into small amplitude signals. In 1987, Blesser and Locanthi firstly discovered the narrow-band dithering [9]. By 1990s, Mahmound Fawzy Wagdy did much research on dithering technology and ADC theoretical analysis including dither can improve the noise of ADC and non-linearity of ADC transfer function [10]-[16]. A growing number of scholars make numerous contributions to improve the performance of $\mathrm{ADC}$, but nobody proposes the optimum general principle for ADC judgments [17]-[23].

Dithering technology is extraordinarily efficient way to make totals errors can be independent with input, and applying dithering is the direct method to obtain these results. A model of non-subtractive dithering, the classical model quantization (CMQ), can be described in Figure 1.

The total errors of this system are defined as the differences between the system output and system input, and are denoted by $\xi=(\boldsymbol{x}+d)^{\prime}-\boldsymbol{x}$. Total errors, without dither added to input signal prior the quantization, can make independence of the input signals are not possible in theoretically because of the inherent nonlinearities of ADC. It is widely used that added dither decrease the relativity in terms of input signals and $\xi$ to improve the dynamic effects of ADC.

This paper discusses the quantization error and dithering. The typical dithering signals are simulated in this paper as follow. Using the simulation to illustrate the results that reconstructed dithering can improve the performance of ADC, compared with traditional typical dithering signal to some extent.

\section{Mathodology}

\subsection{System Modeling}

Dithering, straightforwardly, is a random "noise" process added to a signal prior to its quantization in order to decrease the quantization error and improve the

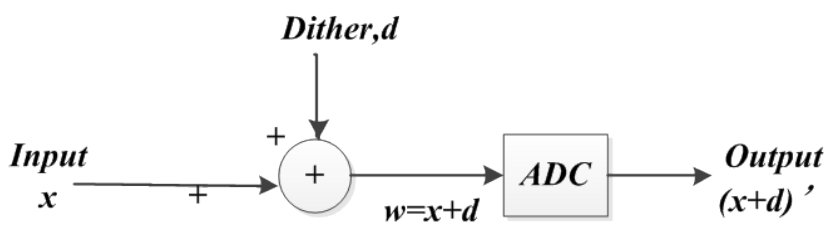

Figure 1. The classical model of quantization (CMQ). 
performance of ADC. The dithering signal generation quantization system is shown in Figure 2. In each model, system inputs are denoted by $x$ and system output by $y$. Thus, $d$ represents the dithering signal in CMQ. We use $w$ to distinguish the quantizer input from system input.

In this particular model, the value of DFT is used to reconstruct the dithering signal. $x(n)$ are the sampled sequence of input signal, and $x q(n)$ represent the quantization result with the dithering signal added zero. Meanwhile, $x(n)$ subtract $x q(n)$ are the value of $v(n)$. The detailed parameters of this model are illustrated in Figure 2 as below.

\subsection{A Condition for Total Error Moments Is Independent with Input}

It has been shown by Wannamaker that total error induced by a non-subtractive dither (NSD) quantization system can be independent with input signal if and only if the dither's characteristic function (CF) or CF (the Fourier transform of its probability density function (PDF) or PDF [24] [25]) obeys a certain condition. Wannamaker's theorem [26] is as follow:

Theorem [26]: In an NSD quantization system, $E\left\{\xi^{l}\right\}$ is independent of the distribution of the system input $x$ for $l=1,2,3, \ldots, m$ if and only if the CF of the dithering satisfied as follow.

$\Phi_{d}^{(i)}(k \Psi)=\mathbf{0} \quad \forall k=Z_{0}, \quad \boldsymbol{i}=\mathbf{0}, \mathbf{1}, 2, \ldots, \boldsymbol{m}-\mathbf{1}$ and $\boldsymbol{m}$ is nonnegative integer. where $\Psi=\frac{2 \pi}{q}$, and $q$ is the step of quantization.

From Wannamaker [26], the statistical analysis of total error $\xi=(x+d)^{\prime}-x$, the difference between system output and system input, can be described as follows.

Now, we define a rectangular window function as

$$
w(x)=\left\{\begin{array}{l}
1,-q / 2 \leq x \leq q / 2 \\
0, \text { elsewhere }
\end{array}\right.
$$

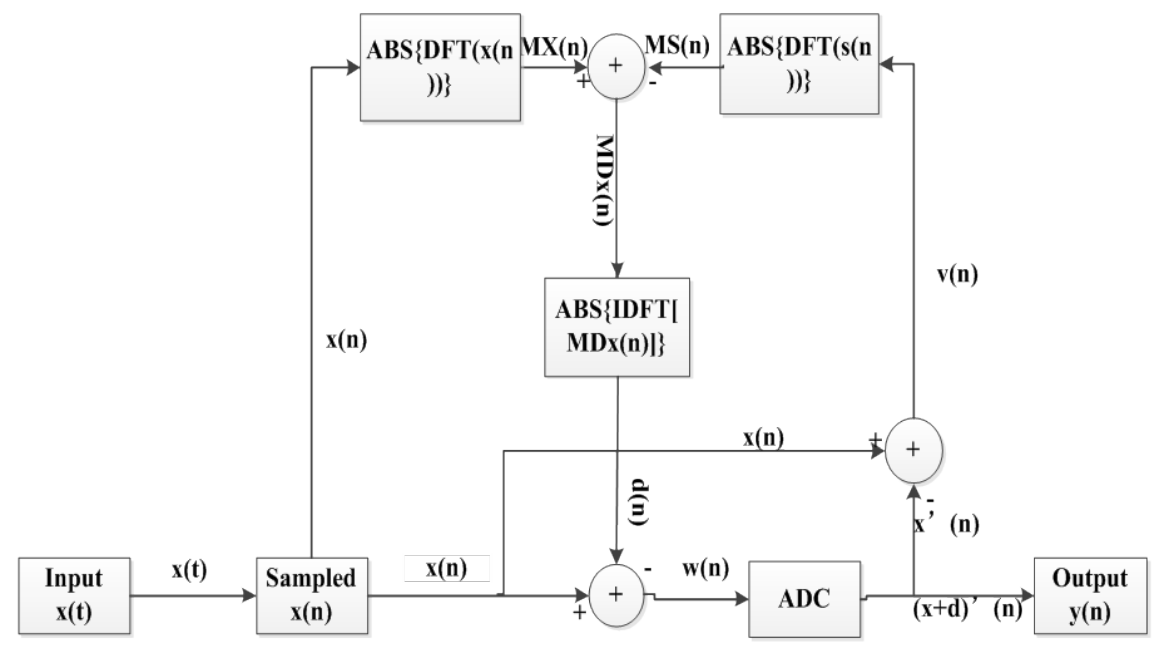

Figure 2. The dithering signal generation quantization system. 
And, an impulse train functions can be defined as

$$
c(x+\xi)=\sum_{k=-\infty}^{\infty} \delta(\xi+x-k q)
$$

The conditional PDF of total error $\xi$ can be calculated as follow:

$$
f_{\xi \mid x}(\xi)=\left(w^{*} f_{d}\right)(\xi) \bullet c(-\xi)
$$

Then, taking the Fourier transform of (3), we find that the CF of $\xi$ is given by

$$
\Phi_{\xi \mid x}(u)=\sum_{l=-\infty}^{\infty} \sin c\left(\frac{q(u-l \Psi)}{2}\right) \Phi_{d}(u-l \Psi)
$$

In this NSD system, we choose the input $x(t)=\sin (w t)$, since an arbitrary signal can be formed by a series of coefficient of Fourier Transform. Then, if the sine function meets the condition of Theorem, any other signals must be satisfied. And the PDF of input is $f_{x}(x)=\frac{1}{\pi \sqrt{1-x^{2}}}$.

$$
\Phi_{x}(u)=\int_{-\infty}^{\infty} f_{x}(x) e^{j u x} d x=\int_{-1}^{1} \frac{1}{\pi \sqrt{1-x^{2}}} e^{j u x} d x
$$

From Figure 2, there is a hysteresis in this system, and a system without dithering added is illustrated in Figure 3.

It is assumed that the interval of input $N$ is big enough, and input $x(n)$ will be independent with $x_{1}(n)=\boldsymbol{x}(n+N)$. The quantization noise $v$ cannot make independence of the input $\boldsymbol{x}(\boldsymbol{n}+N)$, but it is independent with input $\boldsymbol{x}(\boldsymbol{n})$.

Then, the quantization error $v$ can be used with $\boldsymbol{x}$ to reconstruct a dithering based on DFT.

From Bernard Widrow [27], the CF of $v$ can be calculated as follow.

$$
\Phi_{v}(u)=\sum_{l=-\infty}^{\infty} \Phi_{x 1}(l \Psi) \cdot \sin c\left[\frac{q(u+l \Psi)}{2}\right]
$$

From Figure 2, the CF of $v$ multiply the CF of $\boldsymbol{x}$ may constitute the CF of dithering, since $\boldsymbol{v}$ is independence with $\boldsymbol{x}$.

$$
\Phi_{d}(u)=\sum_{l=-\infty}^{\infty} \Phi_{x 1}(l \Psi) \cdot \sin c\left[\frac{q(u+l \Psi)}{2}\right] \bullet \Phi_{x}(u)
$$

According to the theorem [26], $E\{\xi\}$ is independent with any distributed input $\boldsymbol{x}$. And the conditional moments of total errors can be written as follows.

$$
E\left\{\xi^{m} \mid x\right\}=\left.\frac{1}{j^{m}} \Phi_{\xi \mid x}^{(m)}(u)\right|_{u=0}
$$

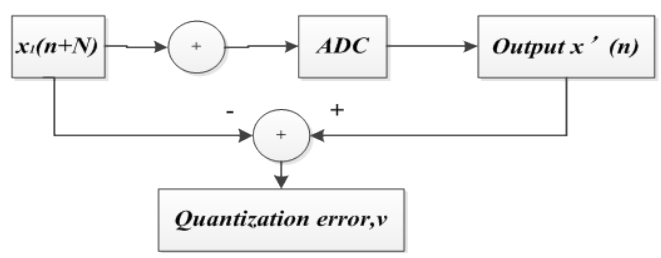

Figure 3. A system with hysteresis. 
We take (7) substitute into (4).

$$
\Phi_{\xi \mid x}(u)=\sum_{l=-\infty}^{\infty} \int_{-1}^{1} \frac{1}{\pi^{2}\left(1-x^{2}\right)} e^{j(l+u) x} d x \bullet \sin c^{2} \frac{u+l \Psi}{2}
$$

From (8) and (9), the first moment of $\xi$, or mean error of $\xi$, is zero for all input signals, which indicate that the ADC can be referred to as a linearized model by use this reconstructed dither. However, the second moments of $\xi$, or error variance of $\xi$, is not zero, indicating that variance is dependent with input. This is sometimes referred to as noise modulation.

\section{Simulation Analysis}

In this simulation, the ADC is 4 bits. The number of the frequency, sample rate, amplitude and numbers of samples in one period for the input signal are respectively 1/40, 5, 1 and 200. The sampled signal through the input signal can avoid aliasing effect. And, this paper use NSD in this system.

The simulation result of traditional dither (Triangular-PDF Dither, GaussianPDF Dither and Uniform-PDF Dither) compared with the reconstructed dither illustrate in Figures 4(a)-(e).

From Figure 4(a), the quantization results of non-dither added loses lots of information compared to original input signal. And this result can decrease the accuracy and the distortion of the signal during its reconstruction Digital-toAnalog Converter (DAC).

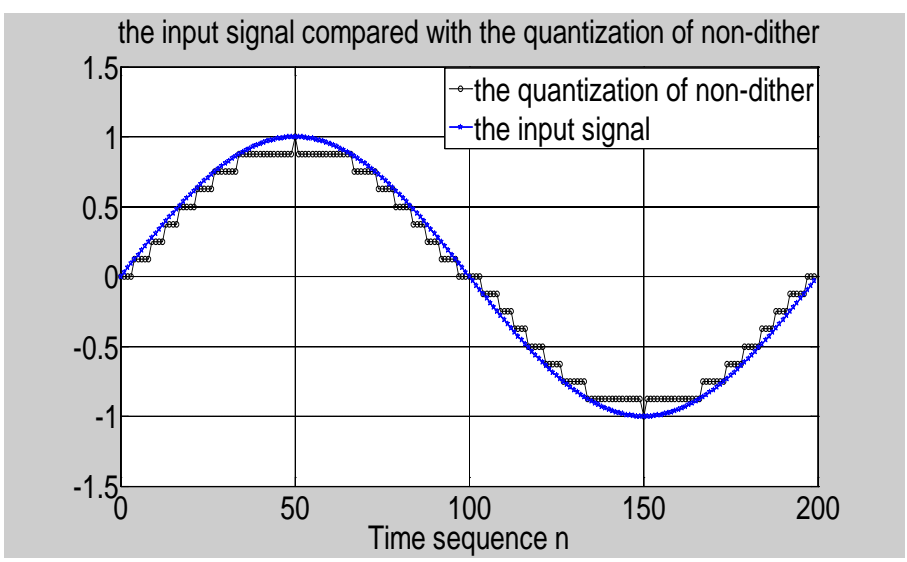

(a)

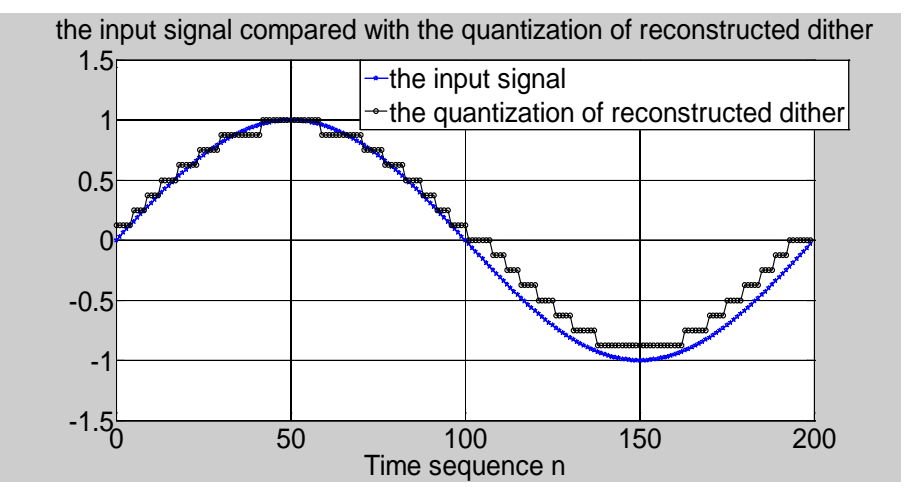

(c) 


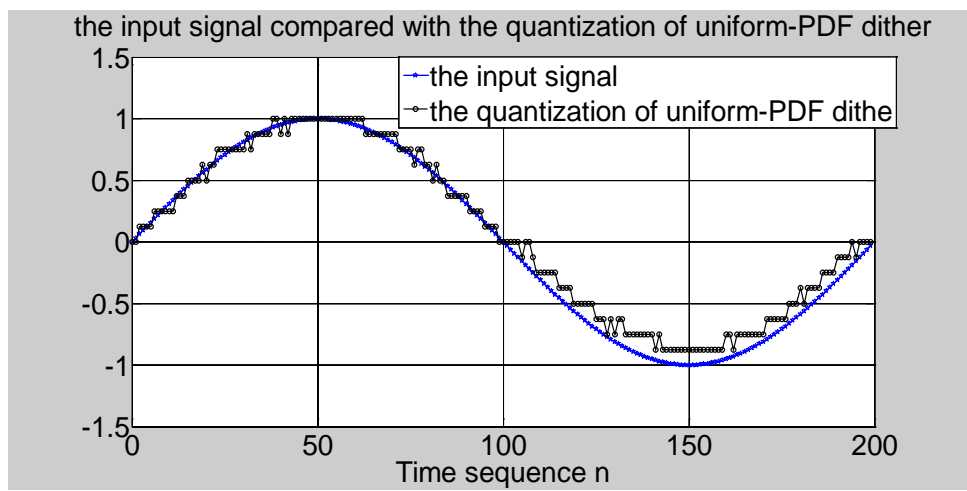

(c)

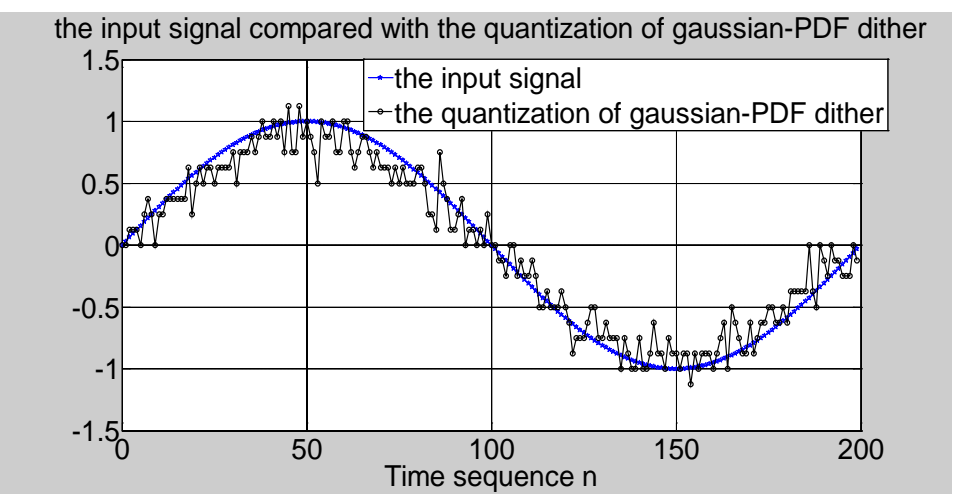

(d)

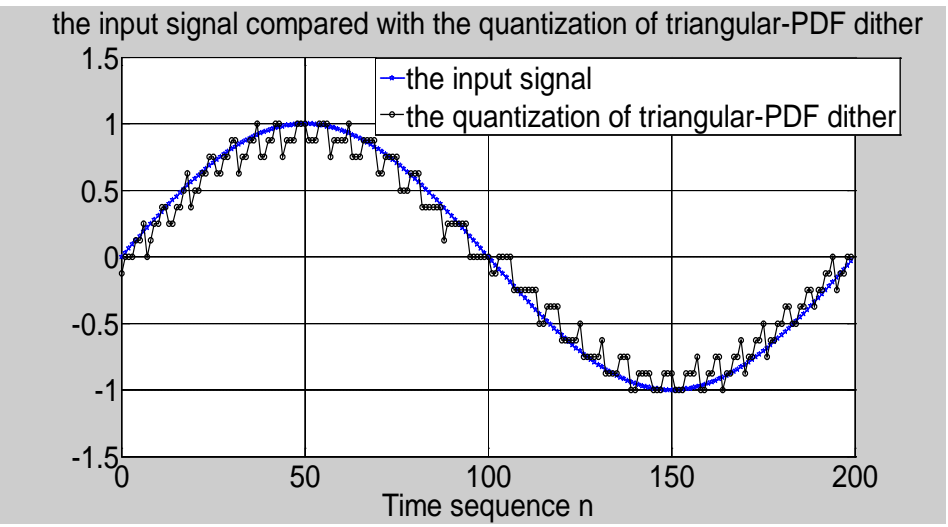

(e)

Figure 4. The quantization results of different dither. (a) The input signal compared with the quantization of non-dither. (b) The input signal compared with the quantization results of reconstructed dither. (c) The input signal compared with the quantization results of uniform-PDF dither. (d) The input signal compared with the quantization results of gaussian-PDF dither. (e) The input signal compared with the quantization results of triangular-PDF dither.

From Figures 4(b)-(e), we can infer that the quantization results of different dither can keep the integrality of the input in some extent. From Figure 4(b) and Figure 4(c), the results curves are smoother than others. There are more signals burr for the result of Gaussian-PDF dither from Figure 4(d). It can be seen that more distortion for the result of triangular-PDF dither from Figure 4(e). 


\section{Conclusion}

In this paper, different dithering have been discussed. And the simulation show that the dithering signal can decrease quantization error and improve the dynamic efficiency of ADC. Moreover, we can see that the effects of the reconstructed dithering are improved compared with others in the performance of ADC. And the accuracies of quantization results for the reconstructing dithering signal are enhanced. Therefore, the application of this reconstructed dithering based on DFT may be used in communication, image and video signal processing.

\section{Acknowledgements}

This work is fully supported by and the Innovation Group Build Plan of Sichuan (No. 2015TD0022), and the Talents Project of Sichuan University of Science and Engineering (No. 2014RC13), and the Opening Project of Artificial Intelligence Key Laboratory of Sichuan Province (2017WZJ01), the Opening Project of Key Laboratory of Higher Education of Sichuan Province for Enterprise Informationalization and Internet of Things (2017WZJ01), and Sichuan University of Science and Engineering talent introduction project (2017RCL11).

\section{References}

[1] Goodall, W.M. (1951) Television by Pusle Code Modulation. Bell Sys. Tech. J., 30, 33-49. https://doi.org/10.1002/j.1538-7305.1951.tb01365.x

[2] Roberts, L.G. (1962) Picture Coding Using Pseudo-Random Noise. IRE Transactions on Information Theory, 8, 145-154. https://doi.org/10.1109/TIT.1962.1057702

[3] Widrow, B. (1960) Statistical Analysis of Ampitude-Quantized Sampled-Data Systems. Trans. Applications and Industry, 79, 555-568.

[4] Schuchman, L. (1964) Dither Signals and Their Effect on Quantizaton Noise. IEEE Transactions on Communication Technology, 12, 162-165. https://doi.org/10.1109/TCOM.1964.1088973

[5] Spang, H.A. and Schultheiss, P.M. (1962) Reduction of Quantizing Noise by Use of Feedback. IRE Transactions on Communications Systems, 10, 373-380. https://doi.org/10.1109/TCOM.1962.1088681

[6] Blesser, B.A. An Investigation of Quantization Noise.

[7] Blesser, B.A. Digitization of Audio: A Comprehensive Examination of Theory, Implementation, and Current Practice.

[8] Vanderkooy, L. On the Audibility of Midrange Phase Distortion in Audio Systems.

[9] Blesser, B. and Locanthi, B. The Application of Narrow-Band Dither Operating at the Nyquist Frequency in Digital Systems to Provide Improved Signal-to-Noise Ratio over Converntional Dither.

[10] Wagdy, M.F. Effect of Various Dither Forms on Quantization Errors of Ideal A/D Converters. IEEE Transactions.

[11] On Instrumentation and Measurement. Vol. 38, No. 4, August 1989, 850-855.

[12] Gray, R.M. (1990) Quantization Noise Spectra. IEEE Transactions on Information Theory, 36, 1220-1244. https://doi.org/10.1109/18.59924

[13] Gray, R.M. and Stockham, T.G. (1993) Dithered Quantizers. IEEE Transactions on Information Theory, 39, 805-812. https://doi.org/10.1109/18.256489 
[14] Carbone, P. and Petri, D. (1994) Effect of Additive Dither on the Resolution of Ideal Quantizers. IEEE Transactions on Instrumentation and Measurement, 43, 389-396. https://doi.org/10.1109/19.293456

[15] Zames, G. and Shneydor, N.A. (1976) Dither in Nonlinear Systems. IEEE Transactions on Automatic Control, 21, 660-667. https://doi.org/10.1109/TAC.1976.1101357

[16] Wagdy, M.F. (1996) Effect of Additve Dither on the Resolution of ADC's with Single-Bit or Mulitbit Errors. IEEE Transactions on Instrumentation and Measurement, 45, 610-615. https://doi.org/10.1109/19.492797

[17] Carbone, P. (1997) Quantitative Criteria for the Design of Dithered-Based Quantizing Systems. IEEE Transactions on Instrumentation and Measurement, 46, 656-659. https://doi.org/10.1109/19.585423

[18] Li, F.L., Duan, J.B. and Wang, Z.H. (2008) A High Linearity 13 Bit Pipelined CMOS ADC. Journal of Semiconductors, 29, 487-501.

[19] Nair, K. and Harjani, R. (2004) A 96 dB SFDR 50 MS/s Digitally Enhanced CMOS Pipeline A/D Converter. IEEE ISSCC Dig. Tech. Papers, San Francisco, 15-19 February 2004, 456-457. https://doi.org/10.1109/ISSCC.2004.1332791

[20] Siragursa, E. and Galton, I. (2004) A Digitally Enhanced 1.8-V 15-Bit 40-M Sample/s CMOS Pipelined ADC. IEEE J. Solid-State Circuits, 39, 2126-2138. https://doi.org/10.1109/JSSC.2004.836230

[21] Liu, H.C., Lee, Z.M. and Wu, J.T. (2005) A 15-b 40-MS/s CMOS Pipelined Analogto-Digital Converter with Digital Background Calibration. IEEE J. Solid-State Circuits, 40, 1047-1056. https://doi.org/10.1109/JSSC.2005.859253

[22] Balestrieri, E., Daponte, P. and Rapuano, S. (2005) A State of the Art on ADC Error Compensation Methods. IEEE Transactions on Instrumentation and Measurement, 54, 1388-1394. https://doi.org/10.1109/TIM.2005.851083

[23] Bogner, P., Kuttner, F., Kropf, C., et al. (2006) A 14 b 100 MS/s Digitally Self-Calibrated Pipelined ADC in 0.13_m CMOS. IEEE ISSCC Dig. Tech. Papers, 2006, 224225.

[24] Lukacs, E. (1960) Characterstic Function. Griffin, London, U.K.

[25] Kawata, T. (1972) Fourier Analysis in Probability Theory. Academic, New York, NY.

[26] Wannamaker, R.A., Lipshitz, S., Vanderkooy, J., et al. (2000) A Theory of Nonsubtractive Dither. IEEE Transactions on Signal Processing, 48, 499-516. https://doi.org/10.1109/78.823976

[27] Widrow, B. and Kollár, I. (2008) Quantization Noise: Roundoff Error in Digital Computation, Signal Processing, Control, and Communications: Preface. Cambridge University Press, Vol. 11, 704-704.

https://doi.org/10.1017/CBO9780511754661 
Submit or recommend next manuscript to SCIRP and we will provide best service for you:

Accepting pre-submission inquiries through Email, Facebook, LinkedIn, Twitter, etc. A wide selection of journals (inclusive of 9 subjects, more than 200 journals)

Providing 24-hour high-quality service

User-friendly online submission system

Fair and swift peer-review system

Efficient typesetting and proofreading procedure

Display of the result of downloads and visits, as well as the number of cited articles Maximum dissemination of your research work

Submit your manuscript at: http://papersubmission.scirp.org/

Or contact ijcns@scirp.org 